\title{
Biotoxicity of heavy metal salts to Bacillus subtilis and their sorption properties
}

\author{
Aleksey Sizentcov ${ }^{1, *}$,Elena Sal'nikova ${ }^{1}$, Elena Barysheva $^{1}$, Yaroslav Sizentcov $^{1}$, and \\ Veronika Sal'nikova ${ }^{1}$ \\ ${ }^{1}$ Orenburg state university, 460018, Prospect Pobedy, 13, Orenburg, Russia
}

\begin{abstract}
The prospect of the use of microorganisms in medical and veterinary practice at the present stage of scientific development has high potential based on natural mechanisms of protection and adaptation of organisms to the effects of adverse factors. The biological adaptation potential of microorganisms to the effects of heavy metals found its practical application in studies of soil and water bioremediation. Based on the above, we set the aim to assess inhibitory characteristics of various heavy metal compounds, the extent of their impact on the growth of populations of probiotic strains Bacillus subtilis, and to study biosorption criteria of the strains. To achieve this aim, we used isolated cultures of probiotic strains B. subtilis 534 and B. subtilis 10641 extracted from drugs Sporobacterin and Vetom 1.1, respectively. Various chemical compounds of iron, copper, zinc, cadmium, and lead with a various anion component were used as growth inhibitors in the study. The criterion for the selection of compounds was a high level of their dissociation in water solutions. Data presented in this work experimentally confirm the capability of using probiotic strains in ecosystems of biological origin. A comparison of two strains of Bacillus subtilis showed a high level of resistance to xenobiotic elements with strain differences in the studied microorganism. The B. subtilis 534 strain revealed a higher level of resistance to compounds with a direct correlation between the inhibitory characteristics of the elements and the level of element sorption from the nutrient substrate.
\end{abstract}

\section{Introduction}

Industrial activity has led to large-scale pollution of the environment with toxic heavy metals and radionuclides. Chemical approaches are available to restore metals, but they are often expensive to use and do not have the specificity necessary to process target metals against the background of competing ions. In addition, such approaches are not applicable to the cost-effective recovery of large-scale underground pollution on the spot $[1,2]$.

Biological approaches offer the potential for highly selective removal of toxic metals combined with considerable operational flexibility; they can be used both in situ and ex situ in different bioreactor configurations. Many of these processes utilize microorganisms, which play a key role in the biogeochemical cycle of toxic metals and radionuclides [3, 4].

\footnotetext{
*Corresponding author: kwan111@yandex.ru
} 
Single or multiple detoxification mechanisms can be applied to any individual metal or group of chemically related metals. However, the type of microorganisms may strongly affect the mechanisms of detoxification. For example, many microbes have specific stress resistance genes that can regulate resistance to toxic metals, and such regulatory genes are centrally positioned on plasmids or chromosomes. In general, the metal resistance regulatory system is based on chromosomal genes, which are more complicated than the plasmid system. On the other hand, systems encoded by plasmids usually interact with the mechanism of the outflow of toxic ions [5].

For many microorganisms, the effectiveness of biological detoxification of metals through targeted microbial activity depends on factors such as the age of culture, the cell shape, $\mathrm{pH}$, contact time, and initial concentrations of metals in the solution [6]. In this regard, several studies with actively growing cells showed that some microorganisms could effectively remove heavy metal ions from the nutrient environment, especially when the concentration of metals was relatively low [7].

Bioaccumulation depends on active metabolic transport through the cell membrane, which can include both irreplaceable and other heavy metals [8].

A number of microorganisms proved that some metal oxyanions got restored by oxidoreductases that were able to knock electrons out of the bacterial transport chain through a pool of quinones [9]. Some of toxic metals can indirectly cause the starvation of microbial cells by pumping electrons out of the respiratory chain [10].

Based on the above, we set the aim to assess inhibitory characteristics of various heavy metal compounds, the extent of their impact on the growth of populations of probiotic strains Bacillus subtilis, and to study biosorption criteria of the strains.

\section{Materials and methods}

To achieve this aim, we used isolated cultures of probiotic strains B. subtilis 534 and B. subtilis 10641 extracted from drugs Sporobacterin and Vetom 1.1, respectively. Various chemical compounds of iron, copper, zinc, cadmium, and lead with a various anion component were used as growth inhibitors in the study. The criterion for the selection of compounds was a high level of their dissociation in water solutions.

The solution to the aim was based on the implication of a number of methodical approaches related to the evaluation of inhibitory characteristics of elements (the diffusion method of agar basins), the degree of influence of elements on the growth of probiotic strains (the colorimetric method), and the study of sorption characteristics of microorganisms (the method of atomic-absorption spectrophotometry).

\section{Results and discussion}

The diffusion method of agar basins combines two methods: the method of basins in the agar layer and the method of serial dilutions [10]. Salts (sulfates, nitrates, chlorides, and acetates) of zinc, copper, iron, cadmium, and lead with high levels of dissociation in water solutions were used as a source of excessive concentrations of cations of essential and xenobiotic elements. The advantage of this method is a visual assessment of the toxicity of chemical compounds in different concentrations under identical conditions, in addition, this method is not only qualitative but is also a quantitative assessment of biotoxicity of chemical compounds under study (Table 1). 
Table 1. Evaluation of inhibitory characteristics of salts of the studied elements on probiotic strains Bacillus subtilis.

\begin{tabular}{|c|c|c|c|c|c|c|}
\hline & \multicolumn{6}{|c|}{ The concentration of salts of metals, $\mathrm{mmol} / \mathrm{ml}$} \\
\hline & \multicolumn{3}{|c|}{ B. subtilis 534} & \multicolumn{3}{|c|}{ B. subtilis 10641} \\
\hline & 0.25 & 0.125 & 0.063 & 0.25 & 0.125 & 0.063 \\
\hline & \multicolumn{6}{|c|}{ Iron compounds } \\
\hline $\mathrm{FeSO} 4$ & $14.3 \pm 0.67$ & $10.0 \pm 0.00$ & $5.0 \pm 0.00$ & $13.7 \pm 0.67$ & $11.0 \pm 1.00$ & $5.0 \pm 0.00$ \\
\hline $\mathrm{Fe}(\mathrm{NO} 3) 2$ & $13.7 \pm 0.58$ & $10.7 \pm 0.58$ & $9.0 \pm 1.33$ & $11.7 \pm 0.29$ & $9.7 \pm 1.61$ & $7.00 \pm 1.32$ \\
\hline \multirow[t]{2}{*}{$\mathrm{FeCl} 3$} & $8.3 \pm 0.88$ & $5.3 \pm 0.33$ & - & $8.3 \pm 0.88$ & $5.0 \pm 0.00$ & - \\
\hline & \multicolumn{6}{|c|}{ Copper compounds } \\
\hline $\mathrm{CuSO} 4$ & $15.7 \pm 4.97$ & $10.3 \pm 3.06$ & $8.0 \pm 2.00$ & $18.7 \pm 1.76$ & $16.7 \pm 0.33$ & $6.67 \pm 2.06$ \\
\hline $\begin{array}{c}\mathrm{Cu}(\mathrm{CH} 3 \mathrm{CO} \\
\mathrm{O}) 2\end{array}$ & $13.7 \pm 3.84$ & $13.0 \pm 3.66$ & $10.0 \pm 2.66$ & $8.1 \pm 2.66$ & $7.7 \pm 2.00$ & - \\
\hline \multirow[t]{2}{*}{$\mathrm{CuCl} 2$} & $22.0 \pm 3.51$ & $19.3 \pm 3.33$ & $12.3 \pm 1.33$ & $20.3 \pm 2.60$ & $15.7 \pm 1.33$ & $10.0 \pm 3.33$ \\
\hline & \multicolumn{6}{|c|}{ Zinc compounds } \\
\hline $\mathrm{ZnSO} 4$ & $24.3 \pm 2.74$ & $22.7 \pm 1.88$ & $19.7 \pm 1.33$ & $20.3 \pm 1.98$ & $12.3 \pm 1.18$ & $1.5 \pm 1.33$ \\
\hline $\begin{array}{c}\mathrm{Zn}(\mathrm{CH} 3 \mathrm{CO} \\
\mathrm{O}) 2\end{array}$ & $28.0 \pm 1.53$ & $25.0 \pm 0.00$ & $22.3 \pm 1.45$ & $26.0 \pm 1.00$ & $24.3 \pm 1.96$ & $19.7 \pm 1.33$ \\
\hline $\mathrm{ZnCl} 2$ & $28.7 \pm 1.33$ & $27.0 \pm 1.00$ & $22.3 \pm 1.45$ & $12.0 \pm 0.66$ & $10.7 \pm 1.33$ & $7.4 \pm 2.88$ \\
\hline \multirow[t]{2}{*}{$\mathrm{Zn}(\mathrm{NO} 3) 2$} & $25.0 \pm 2.50$ & $23.3 \pm 1.21$ & $19.7 \pm 1.88$ & $30.6 \pm 2.06$ & $22.3 \pm 1.17$ & $19.0 \pm 2.66$ \\
\hline & \multicolumn{6}{|c|}{ Cadmium compounds } \\
\hline CdSO4 & $15.0 \pm 0.58$ & $10.7 \pm 0.67$ & $7.7 \pm 2.40$ & $11.7 \pm 0.88$ & $9.3 \pm 0.67$ & $6.0 \pm 1.46$ \\
\hline $\begin{array}{c}\mathrm{Cd}(\mathrm{CH} 3 \mathrm{CO} \\
\mathrm{O}) 2\end{array}$ & $12.7 \pm 0.67$ & $8.0 \pm 1.00$ & $5.8 \pm 1.33$ & $21.7 \pm 1.67$ & $26.7 \pm 2.33$ & $14.0 \pm 3.02$ \\
\hline \multirow[t]{2}{*}{$\mathrm{CdCl} 2$} & $10.00 \pm 1.00$ & $5.3 \pm 1.33$ & - & $28.3 \pm 1.01$ & $22.3 \pm 1.45$ & $21.7 \pm 1.67$ \\
\hline & \multicolumn{6}{|c|}{ Lead compounds } \\
\hline $\mathrm{Pb}(\mathrm{NO} 3) 2$ & $17.0 \pm 0.58$ & $9.7 \pm 0.33$ & $7.0 \pm 0.58$ & $14.7 \pm 0.33$ & $9.7 \pm 0.33$ & $6.7 \pm 0.33$ \\
\hline $\begin{array}{c}\mathrm{Pb}(\mathrm{CH} 3 \mathrm{CO} \\
\mathrm{O}) 2\end{array}$ & $13.3 \pm 0.88$ & $10.3 \pm 0.33$ & $6.7 \pm 0.33$ & $13.0 \pm 0.58$ & $9.7 \pm 0.33$ & $6.7 \pm 0.33$ \\
\hline
\end{tabular}

The colorimetric method was used to determine the effect of salts of the studied elements with a various anion component on the growth of experimental bacterial strains, the need for this study was related to the fact that bacteria are forced not only to search for alternative sources of energy and substrate but also to turn on mechanisms for detoxification of secondary metabolites and other toxigenic factors in the process of growth in batch culture due to the depletion of the nutrient substrate. The assessment of the effects of metals on the growth of the studied microorganisms and the start time of phase $\mathrm{M}$ of growth concentration was carried out by measuring the optical density (OD) of a bacterial suspension at intervals of three hours starting with the background study and continued until three identical values appear, which stated that the Phase $M$ of growth concentration started (Figure 1). To assess the purity of the experiment, the Gram stain was applied at all points of the study. In order to visualize the results of the evaluation of the effects of zinc on the growth of the bacterial strains and the start time of phase $\mathrm{M}$ of growth concentration, we drew diagrams of the growth of the strains where each point represented the average of three measurements in parallel studies.

The atomic absorption method was used to assess the metal ion sorption characteristics of the studied strains (Figure 2). The method is based on the property of atoms of chemical elements formed by spraying ash solutions into acetylene-air flame to absorb light of a certain wavelength. The research was carried out using an atomic-absorption spectrophotometer - an AAS-1-type device with a set of spectral lamps [4].

Experimental data of assessing the inhibitory characteristics of essential and xenobiotic elements in the form of salts with various anion components indicate a relatively high level of resistance of the strains in relation to compounds of iron $\rightarrow$ cadmium $\rightarrow$ lead $\rightarrow$ copper $\rightarrow$ zinc for B. subtilis 534 and iron $\rightarrow$ lead $\rightarrow$ cadmium $\rightarrow$ copper $\rightarrow$ zinc for B. subtilis 10641. It should be noted both strains had resistance to iron chloride and individual 
resistance to cadmium chloride at the concentration of $0.063 \mathrm{mmol} / \mathrm{ml}$ (B. subtilis 534) and copper acetate at $0.063 \mathrm{mmol} / \mathrm{ml}$ (B. subtilis 10641), which indicates not only species resistance of microorganisms, but also a strain one.

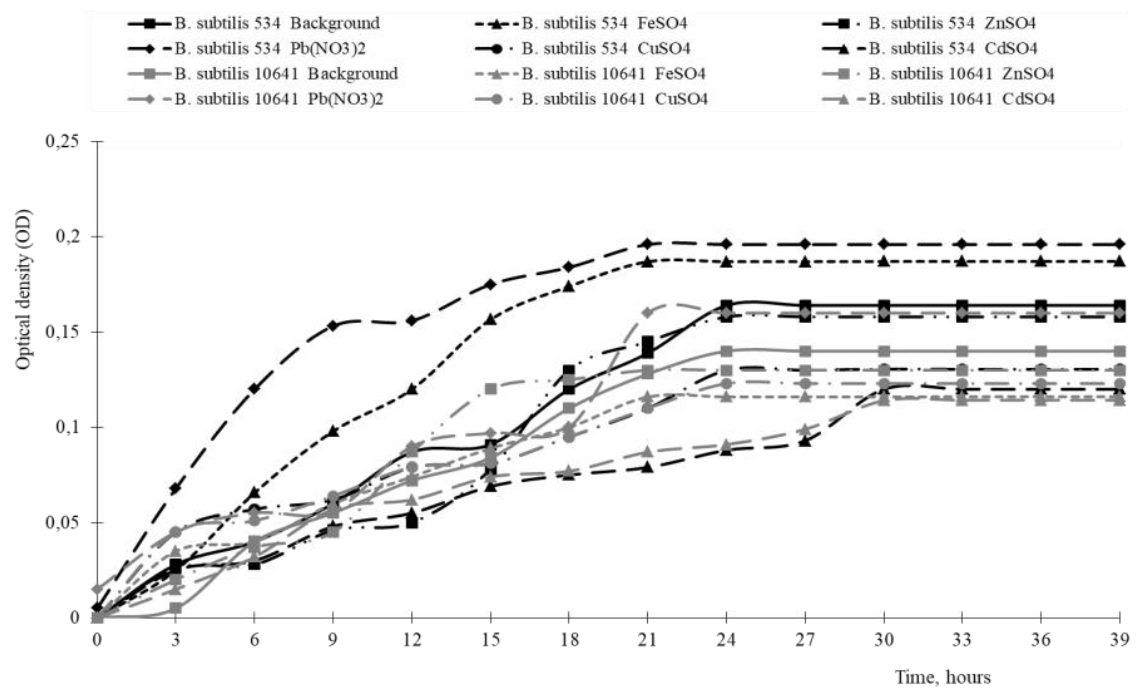

Fig. 1. The effect of the studied elements on the growth of probiotic strains in batch culture.

Studies aimed at assessing the extent of the effect of elements on the growth of populations of the studied microorganisms allow stating the general pattern (Figure 1). The presence of lead and iron cations contributes to an increase in the population density compared to control values whilst there is a reduction in the time of the start of the stationary phase of growth, in our opinion, it is due to biosorption characteristics of microorganisms, as a result of which the density of the population of bacterial culture increases. The most pronounced sub-inhibiting effect on population growth has cadmium it depresses population growth in comparison with control values, but also significantly prolongs the time of the beginning of the stationary phase of growth. 


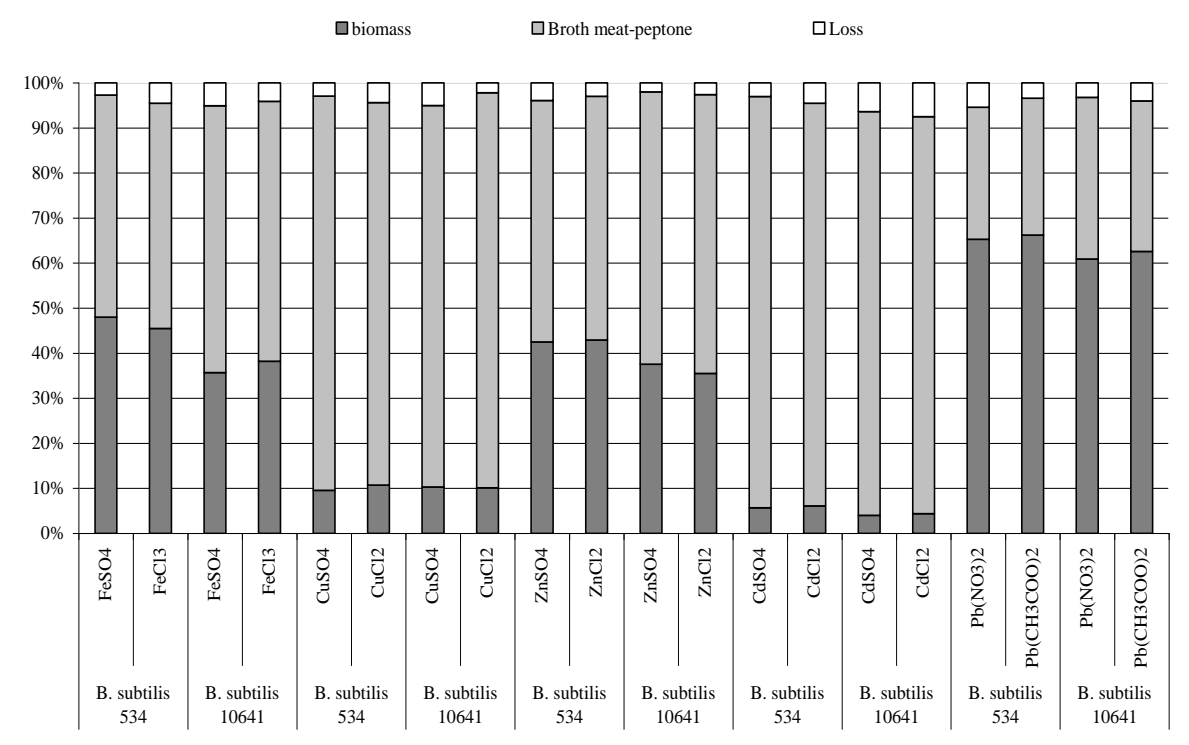

Fig. 2. Assessment of the sorption characteristics of the strains.

Experimental data of biosorption characteristics of the strains (Figure 2) allow stating a direct correlation between the degree of influence of elements on growth and their level of sorption into bacterial biomass. The results show high levels of lead and iron sorption with the highest values for B. subtilis 534 samples. The sorption level for both microorganisms was as follows: lead $\rightarrow$ iron $\rightarrow$ zinc $\rightarrow$ copper $\rightarrow$ cadmium.

\section{Conclusion}

The obtained experimental data show a high level of sorption characteristics of the studied strains of microorganism B. subtilis with revealing common species and strain distinctions of representatives of this species.

Nowadays, there is taking place an active study of the interaction of this genus with heavy metals. Thus, the scientists revealed that the B. cereus RC- 1 strain showed the greatest resistance to $\mathrm{Cd} 2+$ amongst four studied heavy metals. The removal of heavy metals reached saturation within about 13 hours after the incubation and the optimal $\mathrm{pH}$ value was 7.0. The removal efficiency ranges were 14-25\%, 26-42 \%, 35-81\%, and 29-44 $\%$ for $\mathrm{Cu} 2+, \mathrm{Zn} 2+, \mathrm{Cd} 2+$, and $\mathrm{Pb} 2+$, respectively. It was shown that growing cells can accumulate some heavy metals inside the cell, but most get accumulated in the extracellular fraction. The removal of $\mathrm{Cd} 2+$ and $\mathrm{Pb} 2+$ coincided with the takeover of $\mathrm{Na}+$ and $\mathrm{Mg} 2+$, respectively. Thus, B. cereus $\mathrm{RC}-1$ in the growth phase has a high potential for the removal of heavy metals from water solutions, especially $\mathrm{Cd} 2+$. The metal biosorption mechanism observed in this genus is an alternative to chemical precipitation and ultrafiltration, which are used to treat heavy metal contaminations with limited success. Researchers selected three species of Bacillus that were extracted from soils with severe salinity and screened for the detoxification potential of heavy metals, lead, chromium, and copper by biosorption. As a result, bacterial isolates of Bacillus licheniformis NSPA5, Bacillus cereus NSPA8, and Bacillus subtilis NSPA13 showed significant levels of lead biosorption with a maximum of 87-90\% (Bacillus cereus NSPA8). Copper and chromium biosorption was relatively low compared to lead [10]. 
The halo-alkaliphilic species of Bacillus that are present in salty soils produce compatible solvents and exopolymers to survive in the changing conditions of halo-alcans [16]. Thus, these extracellular molecules can offer an adaptive advantage for haloalkaliphilic species of Bacillus in order to effectively tolerate and remove heavy metals through biosorption.

\section{References}

1. A.N. Sizentsov, O.V. Kvan, et al., Life science journal, 11, 18-20 (2014)

2. S. Miroshnikov, A. Kharlamov, et al., Pakistan Journal of Nutrition, 14, 632-636 (2015) http://dx.doi.org/10.3923/pjn.2015.632.636

3. A.N. Sizentsov, O.V. Kvan, et al., Environmental Science and Pollution Research, 25, 15765-15773 (2018) https://doi.org/10.1007/s11356-018-1761-4

4. V. Kalashnikov, A. Zajcev, et al., Environmental Science and Pollution Research, 25, 21961-21967 (2018) https://doi.org/10.1007/s11356-018-2334-2

5. S.A. Peshkov, A.N. Sizentsov, et al., Modern problems of science and education, 4, 17 (2017)

6. G. Sizentsov, T. Karpova, et al., International Journal of GEOMATE, 16, № 55, 8-13 (2019) DOI: $10.21660 / 2019.55 .76923$

7. J. K. Wisch, E. Farrell, et al., Misconceptions and persistence: resources for targeting student alternative conceptions in biotechnology (Biochem Mol Biol Educ, 2018) doi: $10.1002 / \mathrm{bmb} .21176$

8. L. Moro, G. Pezzotti, M. Turemis, et al., Mar. Pollut. Bull., 129(1), 212-221 (2018) doi: 10.1016/j.marpolbul.2018.02.036

9. F. Boriani, N. Fazio, et al., World Neurosurg, 18, 32502-32506 (2018) doi: 10.1016/j.wneu.2018.10.195

10. O. Kvan, G. Duskaev, Sh. Rakhmatullin D. Kosyan, International Journal of Environmental Science and Development, 10(12), 419-423 (2019) DOI: $\underline{10.18178 / \text { ijesd.2019.10.12.1209 }}$ 